\title{
MULTIVARIATE UNIMODALITY
}

\author{
By S. W. Dharmadhikari and Kumar Jogdeo
}

\section{M.S. University of Baroda and University of Illinois}

For univariate distributions there is a generally accepted definition of unimodality due to Khintchine which requires the existence of a number $a$, called vertex, such that the distribution function is convex on $(-\infty, a)$ and concave on $(a, \infty)$. For multivariate distributions, however, unimodality can be defined in several different ways. Anderson (1955) and Olshen and Savage (1970) have given such definitions. This paper first examines the definition which calls a random vector $\left(X_{1}, \cdots, X_{n}\right)$ unimodal if all linear combinations $\sum a_{i} X_{i}$ are univariate unimodal. We show that this definition is somewhat unnatural because the density of such a "unimodal" distribution may not become maximum at the vertex of unimodality. The paper also examines two other definitions based on the results of Sherman (1955). One of these looks at the closed convex hull of the set of all uniform distributions on symmetric convex bodies and the other requires that the probability carried by a symmetric convex set decreases as the set is moved away from the origin in a fixed direction. The equivalence of these definitions was conjectured by Sherman and this paper gives some results having a bearing on this conjecture.

1. Notation and terminology. A set $A \subset R^{n}$ is called symmetric if $\mathbf{x} \in A \Rightarrow$ $(-\mathbf{x}) \in A$. If $A$ and $B$ are subsets of $R^{n}$ and $k \in R$, then $(A+B)$ denotes the set $\{\mathbf{x}+\mathbf{y}: \mathbf{x} \in A$ and $\mathbf{y} \in B\}$ and $k A$ denotes the set $\{k \mathbf{x}: \mathbf{x} \in A\}$. We call a distribution $P$ in $R^{n}$ symmetric if $P(A)=P(-A)$ for all Borel sets $A$ in $R^{n}$. A function $g$ on $R^{n}$ is called symmetric if $g(\mathbf{x})=g(-\mathbf{x})$ for all $\mathbf{x} \in R^{n}$. A set $A \subset R^{n}$ is called star-shaped about $\mathbf{x}$ if, for every $\mathbf{y} \in A$, the line segment $[\mathbf{x}, \mathbf{y}]$ is contained in $A$. A set of distributions in $R^{n}$ is called convex if it is closed under finite mixtures and it is called closed if it is closed in the topology of weak convergence. If $g$ is a nonnegative function on $R^{n}$, then, for every $r>0$, the function $g^{r}$ is defined by $g^{r}(\mathbf{x})=[g(\mathbf{x})]^{r}$. Finally, the abbreviation UM will stand for either "unimodal" or "unimodality."

2. Linear unimodality. We call a random vector $\left(X_{1}, \ldots, X_{n}\right)$ linear $\mathrm{UM}$ about $\mathbf{0}$ if every linear combination $\sum_{1}^{n} a_{t} X_{i}$ has a univariate unimodal distribution about 0 . The set of all distributions in $R^{n}$ which are linear $\mathrm{UM}$ about $\mathbf{0}$ is convex and closed. Moreover, the convolution of two symmetric linear UM distributions in $R^{n}$ is linear UM. However the following example shows that the density of a linear UM distribution may not have a maximum at the mode.

EXAMPLe 2.1. Consider the bivariate density

$$
f(x, y)=k e^{-\left(x^{2}+y^{2}\right) / 2}\left[e^{a\left(x^{2}+y^{2}\right) / 2}-b\right], \quad x \in R, y \in R,
$$

Received April 1974; revised August 1975.

AMS 1970 subject classifications. 52A40, 62E10.

Key words and phrases. Convexity, definitions of multivariate unimodality, convolutions and weak limits of unimodal distributions. 
where $0<a<1, b<1$ and $k$ is so chosen that $f$ is a density. It can be verified that if $b>(1-a)$ then there is a $\delta>0$ such that $f(x, x)$ is strictly increasing in $x \in[0, \delta]$. On the other hand if $b<(1-a)^{\frac{1}{2}}$, then the $x$-marginal of $f$ is unimodal. The circular symmetry of the distribution shows that if $(1-a)<$ $b<(1-a)^{\frac{1}{2}}$, then $f$ is linear $\mathrm{UM}$ about $\mathbf{0}$ but the density is not maximum at $\mathbf{0}$.

3. Central convex unimodality and monotone unimodality. Two definitions of multivariate unimodality, applicable to symmetric distributions, can be based on the results of Sherman (1955).

Definition 3.1. A distribution $P$ in $R^{n}$ is called central convex $\mathrm{UM}$ if it in the closed convex hull of the set of all uniform distributions on symmetric compact convex bodies in $R^{n}$.

Definition 3.2. A distribution $P$ in $R^{n}$ is called monotone $\mathrm{UM}$ if for every symmetric convex set $C \subset R^{n}$ and every nonzero $\mathbf{x} \in R^{n}$, the quantity $P(C+k \mathbf{x})$ is nonincreasing in $k \in[0, \infty]$.

Clearly a central convex UM distribution is symmetric. The same is true of monotone UM distributions.

Theorem 3.1. A monotone UM distribution in $R$ is symmetric and unimodal. A monotone UM distribution in $R^{n}$ is symmetric.

Proof. Let $F$ be the distribution function of a monotone UM distribution $P$ in $R$. Then for $x \geqq 0$ and $h>0$,

or

$$
F(x+h)-F(x) \geqq F(x+2 h)-F(x+h)
$$

$$
F(x+h) \geqq \frac{1}{2}[F(x)+F(x+2 h)] .
$$

Thus $F$ is concave on $[0, \infty)$. Similarly $F$ is convex on $(-\infty, 0]$. Hence $F$ is unimodal. Further the derivatives $F^{\prime}(x)$ and $F^{\prime}(-x)$ exist simultaneously for all $\mathbf{x} \in A$, where the Lebesgue measure of $A^{c}$ is zero. If possible, suppose that there is an $x \in A$ such that $F^{\prime}(x)>F^{\prime}(-x)$. We may assume that $x>0$. Then there is a $\delta \in(0, x)$ such that $P(x, x+\delta]>P(-x,-x+\delta]$. Adding $P(-x+\delta, x]$ to both sides we get

$$
P(-x+\delta, x+\delta]>P(-x, x],
$$

which contradicts the monotone UM of $P$. Hence $F^{\prime}(x)=F^{\prime}(-x)$ for all $x \in A$. This proves that $P$ is symmetric. Now suppose that $\mathbf{X}=\left(X_{1}, \cdots, X_{n}\right)$ has a monotone UM distribution. Then it is easy to show that the distribution of each linear combination $\sum_{1}^{n} a_{\imath} X_{i}$ is monotone $\mathrm{UM}$ and hence symmetric. It follows that $\mathbf{X}$ has a symmetric distribution. This proves the theorem.

It is clear from the very definition that the set of all central convex UM distributions in $R^{n}$ is closed and convex. Similarly the set of all monotone UM distributions in $R^{n}$ is convex. We show below (Theorem 3.2) that this set is also closed. We omit the proof of the following lemma. 
Lemma 3.0. Let $\mu$ be a Borel measure in $R^{n}$. Then, for every convex Borel set $C$ in $R^{n}$,

$$
\mu(C)=\sup \{\mu(D): D \subset C \text { and } D \text { is compact convex }\} .
$$

Moreover, if $C$ is symmetric, then the supremum can be taken over symmetric, compact, convex subsets $D$ of $C$.

Corollary 3.1. A distribution $P$ in $R^{n}$ is monotone UM if, and only if, the property mentioned in Definition 3.2 holds for all symmetric compact convex bodies $C \subset R^{n}$.

THEOR EM 3.2. Suppose $\left\{P_{m}, m \geqq 1\right\}$ is a sequence of monotone UM distributions in $R^{n}$, and let $P_{m} \rightarrow P$ weakly. Then $P$ is monotone UM.

Proof. Let $C$ be a symmetric compact convex body in $R^{n}$ and let $\mathbf{x} \in R^{n}$ be nonzero. We have to show that $P(C+k \mathbf{x})$ is nonincreasing in $k \in[0, \infty)$. For $k \geqq 0$ and $\alpha>0$, write $D(k, \alpha)=\alpha C+k \mathbf{x}$. Since $C$ is a symmetric convex body, for fixed $k$ and varying $\alpha$, the boundaries of $D(k, \alpha)$ are disjoint. Therefore the set

$$
A_{k}=\{\alpha>0: P[\text { Boundary of } D(k, \alpha)]>0\}
$$

is countable. Suppose now that $0 \leqq k<l<\infty$ and $\alpha \in\left(A_{k} \cup A_{l}\right)^{C}$. Then the boundaries of both $D(k, \alpha)$ and $D(l, \alpha)$ have $P$-measure zero. Therefore, by weak convergence

$$
P_{m}[D(k, \alpha)] \rightarrow P[D(k, \alpha)] \quad \text { and } \quad P_{m}[D(l, \alpha)] \rightarrow P[D(l, \alpha)] .
$$

Since $P_{m}$ is monotone UM, $P_{m}[D(k, \alpha)] \geqq P_{m}[D(l, \alpha)]$. Therefore (3.1) implies that $P[D(k, \alpha)] \geqq P(D(l, \alpha)]$. Letting $\alpha \rightarrow 1$ from above through the dense set $\left(A_{k} \cup A_{l}\right)^{C}$ and using the fact that $C$ is compact, we see that $P[C+k \mathbf{x}] \geqq$ $P[C+l \mathbf{x}]$. This completes the proof of the theorem.

The following extension of a result of Anderson (1955) was proved by Sherman (1955).

THEOREM 3.3. Every central convex UM distribution is monotone UM.

Sherman conjectured that the converse of Theorem 3.3 is true and also proved that the set of all central convex UM distributions in $R^{n}$ is closed under convolutions. Therefore, if Sherman's conjecture is true, then the convolution of a monotone UM distribution with a central convex UM distribution would be monotone UM. This consequence is verified by Theorem 3.4.

In what follows we use $V_{n}$ to denote Lebesgue measure in $R^{n}$. Lemma 3.1, below, follows immediately from definitions. Lemma 3.2 is due to Sherman (1955).

Definition 3.3. A nonnegative function $g$ on $R^{n}$ is called convex UM if, for every $c>0$, the set $\{\mathbf{x}: g(\mathbf{x})>c\}$ is convex.

LEMMA 3.1. If $g$ is a nonnegative function on $R^{n}$ which is concave on its support, then, for every $r>0$, the function $g^{r}$ is convex UM. 
Lemma 3.2. (Sherman). Let $C$ and $D$ be compact convex sets in $R^{n}$ and let $\Psi(\mathbf{x})=V_{n}[C \cap(D+\mathbf{x})]$. Then $\Psi^{1 / n}$ is concave on its support and hence $\Psi$ is convex UM.

LEMMA 3.3. Every marginal of the uniform distribution on a compact convex body in $R^{n}$ has a convex UM density which is continuous on the interior of its support.

Proof. Write $R^{n}=\Omega_{1} \times \Omega_{2}$, where $\Omega_{1}=R^{m}, \Omega_{2}=R^{n-m}$ and $m<n$. Let $P$ denote the uniform distribution on a compact convex body $C$ in $R^{n}$. The marginal of $P$ in $\Omega_{1}$ will be denoted by $P_{1}$. Let $D_{1} \subset \Omega_{1}$ and $D_{2} \subset \Omega_{2}$ be compact convex bodies and write $D=D_{1} \times D_{2}$. By Lemma 3.2

$$
P^{1 / n}(D+\mathbf{z})=\frac{V_{n}^{1 / n}[C \cap(D+\mathbf{z})]}{V_{n}^{1 / n}(C)}, \quad \quad \mathbf{z} \in R^{n}
$$

is concave on its support, namely $C-D$. Letting $D_{2} \rightarrow \Omega_{2}$ we see that $P_{1}^{1 / n}\left(D_{1}+\mathbf{x}\right)$ is concave on its support, namely, $C_{1}-D_{1}$, where $C_{1}$ is the projection of $C$ on $\Omega_{1}$. From Chapter IV, Section 6 of Saks (1964), we know that a density $f_{1}$ of $P_{1}$ is given by

$$
f_{1}(\mathbf{x})=\lim \inf _{D_{1} \rightarrow\{0\}}\left[P_{1}\left(D_{1}+\mathbf{x}\right) / V_{m}\left(D_{1}\right)\right], \quad \mathbf{x} \in \Omega_{1} .
$$

Since the lim inf of a sequence of concave functions is concave we see that $f_{1}^{1 / n}$ is concave on its support $C_{1}$. Now Lemma 3.1 shows that $f_{1}$ is convex UM. The continuity of $f_{1}^{1 / n}$ and hence of $f_{1}$ on the interior of $C_{1}$ follows from its concavity [see Theorem 10.1 of Rockafellar (1970)]. This proves the theorem.

LeMma 3.4. If $P$ is a monotone $\mathrm{UM}$ distribution in $R^{n}$, $h$ is a symmetric convex $\mathrm{UM}$ function on $R^{n}$ and $\mathbf{y} \in R^{n}$ is nonzero, then $\int h(\mathbf{x}+k \mathbf{y}) d P(\mathbf{x})$ is nonincreasing in $k \in[0, \infty)$.

Proof. The lemma follows by the standard method of first verifying it for indicators of symmetric convex sets and then extending it to general symmetric convex UM functions by linearity and the monotone convergence theorem.

Theorem 3.4. Let $P$ and $Q$, respectively, be monotone $\mathrm{UM}$ and central convex $\mathrm{UM}$ distributions in $R^{n}$. Then the convolution $P * Q$ is monotone UM.

Proof. Let $Q_{B}$ denote the uniform distribution on a symmetric compact convex body $B \subset R^{n}$. The theorem will follow if we prove that $P * Q_{B}$ is monotone UM.

Apart from a constant multiplier, a density $f_{B}$ of $\left(P * Q_{B}\right)$ is given by $f_{B}(\mathbf{y})=$ $P(B+\mathbf{y})$. Let $D \subset R^{n}$ be a symmetric compact convex body and let $\mathbf{x} \in R^{n}$ be nonzero. The

$$
\left(P * Q_{B}\right)(D+k \mathbf{x})=\int_{D+k \mathbf{x}} f_{B}(\mathbf{y}) d \mathbf{y}=\int_{D} P(B+\mathbf{y}+k \mathbf{x}) d \mathbf{y} .
$$

Let $\left\{P_{m}, m \geqq 1\right\}$ be a sequence of absolutely continuous distributions in $R^{n}$ covverging weakly to $P$. Let $g_{m}$ be a density of $P_{m}$. Then (3.2) shows that

$$
\left(P_{m} * Q_{B}\right)(D+k \mathbf{x})=\int_{D} \int_{B} g_{m}(\mathbf{y}+\mathbf{z}+k \mathbf{x}) d \mathbf{z} d \mathbf{y} .
$$


Suppose $Y$ and $Z$ are independently and uniformly distributed on $D$ and $B$ respectively. Then the right side of (3.3) equals $E\left[g_{m}(\mathbf{Y}+\mathbf{Z}+k \mathbf{x})\right]$. By a standard theorem this expectation can be computed by using the density $h_{B}(\mathbf{u})$ of $\mathbf{U}=$ $\mathbf{Y}+\mathbf{Z}$. Therefore

$$
\left(P_{m} * Q_{B}\right)(D+k \mathbf{x})=\int g_{m}(\mathbf{u}+k \mathbf{x}) h_{B}(\mathbf{u}) d \mathbf{u},
$$

or

$$
\left(P_{m} * Q_{B}\right)(D+k \mathbf{x})=\int h_{m}(\mathbf{u}-k \mathbf{x}) d P_{m}(\mathbf{u}) .
$$

We want to show that (3.4) holds with $P_{m}$ replaced by $P$, at least for enough choices of $B$. Now $\left(P_{m} * Q_{B}\right)$ converges weakly to $\left(P * Q_{B}\right)$. Further, since $\left(P * Q_{B}\right)$ is absolutely continuous, $(D+k \mathbf{x})$ is a continuity set of $\left(P * Q_{B}\right)$. Hence the left side of (3.4) converges to $\left(P * Q_{B}\right)(D+k \mathbf{x})$. By Lemma 3.3, the discontinuity points of $h_{B}(\mathbf{u}-k \mathbf{x})$ are all on the boundary of $B+D+k \mathbf{x}$. Now, for varying $\alpha>0$, the boundaries of $\alpha B+D+k \mathbf{x}$ are disjoint. Therefore, there is a dense set $A$ of $\alpha$ 's for which $(\alpha B+D+k \mathbf{x})$ is a continuity set for $P$. It follows from the well-known theorem of Mann and Wald (1943) that, for $\alpha \in A$,

$$
\int h_{\alpha B}(\mathbf{u}-k \mathbf{x}) d P_{m}(\mathbf{u}) \rightarrow \int h_{\alpha B}(\mathbf{u}-k \mathbf{x}) d P(\mathbf{u}) .
$$

Therefore, for $\alpha \in A$

$$
\left(P * Q_{\alpha B}\right)(D+k \mathbf{x})=\int h_{\alpha B}(\mathbf{u}-k \mathbf{x}) d P(\mathbf{u}) .
$$

Now $P$ is monotone UM and, by Lemma $3.3, h_{\alpha B}$ is symmetric and convex UM. Therefore (3.5) and Lemma 3.4 show that $\left(P * Q_{\alpha B}\right)$ is monotone UM whenever $\alpha \in A$. It follows by continuity that $\left(P * B_{B}\right)$ is monotone UM. The proof of the theorem is complete.

COROllary 3.2. Every monotone UM distribution is the weak limit of absolutely continuous monotone UM distributions.

Proof. Consider $\left(P * Q_{B}\right)$ of the preceding proof and let $B \rightarrow\{\mathbf{0}\}$.

4. Miscellaneous results and open questions. For comparative purposes we give the following definition.

Definition 4.1. The distribution of a random vector $\mathbf{x}=\left(X_{1}, \cdots, X_{n}\right)$ is said to be star UM about $\mathbf{0}$ if it is $n$-unimodal in the sense of Olshen and Savage (1970), that is, if $t^{n} E[g(t \mathbf{X})]$ is nondecreasing in $t \in[0, \infty)$ for every nonnegative, bounded, Borel-measurable function $g$.

The reason for the term star UM is that if $f$ is the density of an absolutely continuous distribution in $R^{n}$ which is star UM about $\mathbf{0}$, then, for every $c>0$, the set $\{\mathbf{x}: f(\mathbf{x})>c\}$ is star-shaped about $\mathbf{0}$ [see Theorem 6 and Corollary 3 of Olshen and Savage (1970)].

Example 2.1 shows that a linear UM distribution need not be star UM. It is also easy to show that a star UM distribution need not be linear UM. Thus there is no implication relationship between star UM and linear UM. 
THEOREM 4.1. Every monotone UM distribution is linear UM and star UM.

Proof. Suppose $P$ is a monotone UM distribution in $R^{n}$. Let $Q_{\delta}$ denote the uniform distribution on the sphere $C_{\delta}$ in $R^{n}$ with centre $\mathbf{0}$ and radius $\delta$. Apart from a constant multiplier, a density $f_{\delta}$ of $\left(P * Q_{\delta}\right)$ is given by $f_{\delta}(\mathbf{x})=P\left(C_{\delta}+\mathbf{x}\right)$. The monotone UM property of $P$ implies that $f_{\delta}(k \mathbf{x})$ is nonincreasing in $k \in[0, \infty)$ for every fixed nonzero $\mathbf{x} \in R^{n}$. Thus $f_{\delta}$ is star UM. Letting $\delta \rightarrow 0$ we find that $P$ is star UM. The proof that $P$ is also linear UM is straightforward and is omitted.

REMARK. If a density $f$ on $R^{n}$ has spherical symmetry, that is, $f(\mathbf{x})$ depends on $\sum x_{i}{ }^{2}$ alone, then star UM, monotone UM and central convex UM of $f$ are all equivalent. However, we cannot include linear UM in this list in view of Example 2.1.

REMARK. The properties of linear UM, monotone UM and central convex UM of a distribution are inherited by its marginals. This result is trivial for linear UM, follows from Lemma 4.3 for central convex UM and is easy to show for monotone UM. The marginals of a star UM distribution need not be star UM. Das Gupta (1974) has given an example where a marginal of an absolutely continuous symmetric convex unimodal distribution does not have a convex unimodal density (see Definition 3.3).

EXAMPle 4.1. This example might disprove Sherman's conjecture. Let $A_{1}, \cdots, A_{6}$ be the vertices of a regular hexagon centered at the origin. Let $T_{1}, T_{2}$, respectively, be the triangles $A_{1} A_{3} A_{5}$ and $A_{2} A_{4} A_{6}$. The set $T_{1} \cup T_{2}$ consists of six outer triangles and an inner hexagon. Suppose $P$ is a distribution with support $T_{1} \cup T_{2}$ and having density $\alpha$ on the outer triangles and $\beta$ on the inner hexagon. Suppose $2 \alpha \leqq \beta<3 \alpha$. Then it can be shown that $P$ is not central convex UM. It is, however, believed that $P$ is monotone UM. Even if this belief turns out to be wrong, we would still get a symmetric star UM and linear UM distribution which is not monotone UM.

Open questions which are of interest are to decide whether (i) monotone $\mathrm{UM} \Rightarrow$ central convex UM; (ii) symmetry plus star $\mathrm{UM}$ plus linear $\mathrm{UM} \Rightarrow$ monotone UM; or (iii) the set of all monotone UM distributions in $R^{n}$ is closed under convolutions.

Acknowledgment. The authors are grateful to the referees for useful comments on the original draft of this paper.

\section{REFERENCES}

[1] Anderson, T. W. (1955). The integral of a symmetric unimodal function. Proc. Amer. Math. Soc. 6 170-176.

[2] Das Gupta, S. (1974). A generalization of Anderson's theorem on unimodal functions. Technical Report 133, Stanford Univ.

[3] Mann, H. B. and Wald, A. (1943). On stochastic limit and order relationships. Ann. Math. Statist. 14 217-226. 
[4] Olshen, R. A. and Savage, L. J. (1970). Generalized unimodality. J. Appl. Probability 7 21-34.

[5] Rockafellar, R. T. (1970). Convex Analysis. Princeton Univ. Press.

[6] SAKs, S. (1964). Theory of the Integral, 2nd revised ed. Dover, New York.

[7] Sherman, S. (1955). A theorem on convex sets with applications. Ann. Math. Statist. 26 763-767.

Department of Statistics

M.S. UNIVERSITY OF BARODA

BARODA 2, INDIA
Department of Mathematics UNIVERSITY OF ILLINOIS

URBANA, ILLINOIS 61801 